\title{
Control of mechatronic drives considering the type and number of the nonlinearities of a mechanical converter
}

\author{
Ricardo Tellez, Valentin Morozov, Alexey Zhdanov, Elena Novikova and Dmitryi Gavrilov
}

Department of the mechanical engineering, Vladimir State University, Gorky str. 87, Vladimir, Russia

\begin{abstract}
In this article the particularities of the dynamics study of mechatronic drives that operate at low velocities are discussed; the origins of frictional self-exited oscillations to develop an algorithm for nonlinearity correction are analyzed; a drive mathematical model and the qualitative picture of the self-exited oscillations regimes were presented for the three cases: a "system with clearance and friction-free"; a system without clearance with "dry friction" and a system with clearance and "dry friction". Therefore, an algorithm to compensate the frictional self-excited oscillations based on the feedback introduction for the sliding velocity of the linear motion output link was proposed.
\end{abstract}

\section{Introduction}

The concept of designing an electromechanical drive as a single mechatronic device is widely known and has been realized during the creation of a mechatronic single-block drive, that is a whole structure based on a contactless torque motor to the hollow rotor built with a planetary screw or roller screw mechanism which provides either a linear or rotational output motion Fig. 1 [1-4]. The software implementation and hardware execution of the motion controller by the drives largely determine the quality of the output motion [5-8].

The single-block design which distinguishes by its compactness, increased stiffness, reliability, energyefficiency and a lower vibroactivity [3] is engineeringly manufacturable and can be unified with other elements. The transition to the single-block principle of construction provides an improvement of the drive operation and control, an increased reliability, even during failure of individual elements, a reduction in time and cost to get the object being controlled ready for operation, and a mass large saving, which is reduced not only by a reduction in the mass of the drives themselves, but due to the reduction of auxiliary fixing units and mechanically connected blocks. The use of self-locking actuators ensures a reliable neutralization of the failure effects of locking devices [9-10].

The design of mechatronic actuators which operate at low velocities is not possible without knowing the dynamic, computable and other functioning regularities of its actuating elements. In this sense, the study of the mechatronic modules dynamics seems to be an inherent stage in the design of modern systems for the implementation of a time-optimal motion control algorithm [5, 6]. One of the most difficult obstacles to eliminate for ensuring the specified quality is the manufacturing and wear errors, and, therefore, the clearance in the transmission and its kinematic and static mechanical properties instability. The instability of the specified output displacement principle can also be caused by factors such as self-locking in the transmission, control torque pulsations and load oscillations which are caused by nonlinear frictional properties and a high drive vibroactivity.

\section{Materials and methods}

The enhancement of the functionality and the improvement of the dynamic mechanical properties of electromechanical drives is possible only on the basis of a new approach to their design and development. In this case, in addition to the kinematic requirements, the dynamic ones are brought to the forefront. It is necessary to consider the dynamic interaction of the actuating mechanism and the electric motor.

To make the electromechanical drive the source of a smooth and uniform motion, it is necessary to minimize the causes of irregular running in the mechanical transmission and in the engine. No linear motion drive can provide an "ideal" smooth and uniform motion. All the time there are some external perturbing influences that generate an irregular stroke running of the actuating body. This is like the disturbances caused by the electric

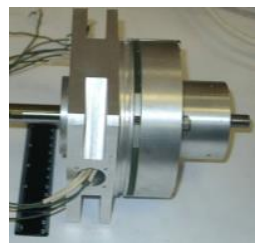

a)

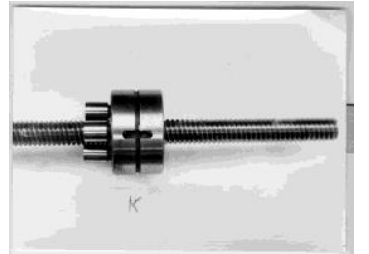

b)
Fig. 1. a) External view of the mechatronic electromechanical module with an incorporated roller screw mechanism. b) Roller screw mechanism 
motor, and the disturbances caused by the actuating mechanism and the working process executed in the machine.

It is assumed that the mechanical drive system has an infinitely high stiffness, and the entire inertia of the electromechanical system is concentrated on the motor shaft as the given moment of inertia. Increasing it, we reduce the motion deviation magnitude of the uniform motion. In the practice, this is usually achieved by installing on the output engine shaft an additional mass, i.e. a flywheel which has a large given torque.

The rotation unevenness of the output link is dangerous, because it causes loads in the transmission mechanism, in addition, it leads to the appearance of a variable component of the driving torque, which can adversely affect the engine operation and cause a reduction in its efficiency.

By installing the flywheel on the output engine shaft, we reduce the torque portion that is associated with the disturbance from the engine. This increases the load caused by the disturbance from the actuator. This can be avoided by installing the flywheel on the output shaft of the transmission device. However, this way is generally unacceptable, since the output shaft is running at low velocity and the proper moment of inertia of the flywheel mounted on it must be i2 times greater than the moment of inertia, given to the motor shaft. The installation of such a large flywheel mass is most often unprofitable for design reasons. The influence of the flywheel on the dynamic loads of the drive, on the one hand, is positive, but on the other the installation of additional mass leads to the increment of the rotation unevenness.

The problem to chose the location of the flywheel mass for a drive traditionally actuating (the engine is the actuator) with high requirements for the smoothness of the output displacement is very delicate. In order to achieve a smooth displacement of the actuating mechanism by its additional weighting, we are obliged to operate the engine at a larger value of the rating torque due to the permissible overload. It is possible to eliminate this negative phenomenon only by using special methods (forcing the engine by increasing the voltage, torque and similar techniques; covering the engine with a tachometer feedback; using engines with increased sliding motion or load regulators). In the end, the aim to eliminate the noted limitations of the flywheel electromechanical drive will lead to a significant increment of its dimensions.

So, the physically existing inertia can be eliminated only by removing an element from the drive that has this inertia, which is not always feasible. But it is possible to compensate the drive inertia influence, using its special arrangement, without involving additional masses.

The DC motors of the DBM series are well known, whose design allows to place the actuator inside the hollow rotor. Thus, the electromechanical drive takes the monoblock form: The motion source (engine) is as close as possible to the actuating link. As a result, the quality of the output displacement will be mainly determined by the mechanical part of the drive; and the rotor rotation unevenness will be partially "smoothed out" (or "damped") by its weighting with the mechanical transmission. This design peculiarity should reduce the load and torque variables in the actuating mechanism and the engine; and stabilize the drive's output motion. In addition, the transmission mechanism is simultaneously an actuator: there is a significant reduction in the kinematic chain

\subsection{Problem formulation}

In an electromechanical drive which operates at low velocities the frictional self-excited oscillations (SEO) arise. The presence of clearance in the transmission also leads to self-excited oscillations. The analysis of the selfexcited oscillating regimes is a necessary stage for the development of a microprocessor algorithm to correct the nonlinearity.

The compensation of the clearance negative influence can be carried out by introducing a correcting link or using a microprocessor control (a software). The use of a microprocessor control provides increased opportunities to compensate the clearance influence on the drive quality indices. A significant advantage of this compensation method is the relative simplicity of the software implementation.

The task of the control in the system with clearance is to get fastest backlash elimination in such a way that at the moment of the connection the links do not impact each other as a result of this elimination. That is more strictly formulated as follows. Let us assume that at the disconnection time, the 1 st link was in the $\left(x_{1}^{0}, v_{1}^{0}\right)$ state, and the 2 nd link was in the $\left(x_{2}^{0}, v_{2}^{0}\right)$ state. It is required to find such a trajectory that translates the system in the shortest time $\tau$ from the initial state $\left(x_{1}^{0}, v_{1}^{0}\right)$ to the new state $\left(x_{2}^{0}+v_{2}^{0} \tau \pm \Delta_{J}, v_{2}^{0}\right)$ (the sign " \pm " is chosen depending on the sign of the control signal at the disconnection time; to determine it, we will consider the signal positive). The coordinates of the new state correspond to the position in which the 2 nd link will move in the time $\tau$, and the 1 st link should be transferred to the same state. The necessity of a high-speed response ( $\tau \rightarrow \min )$ means that the control signal should be the maximal according to the module $\left(\left|u_{\text {упр }}\right|=U_{0}\right)$, and the number of switchings should be the minimal possible, i.e. one switch or no switching at all. In general, switching is required, and the optimal trajectory consists of two sections: the engine speeding-up by the signal $u_{\text {упр }}=U_{0}$ before the switching time $\tau *$ and the engine locking by the opposite connection $u_{\text {упр }}=-U_{0}$ before the backlash elimination end time $\tau$.

\subsection{Mathematical model}

The dynamics of the servo drive which consists of a brushless DC electric motor and a roller screw actuating mechanism (RSM) is described by a two-mass model. The first mass is rigidly connected to the motor shaft and is described by the phase variables $x_{1}$ and $v_{1}=\dot{x}_{1}$; the state of the second mass (load) is determined by the variables $x_{2}$ and $v_{2}=\dot{x}_{2}$ : 


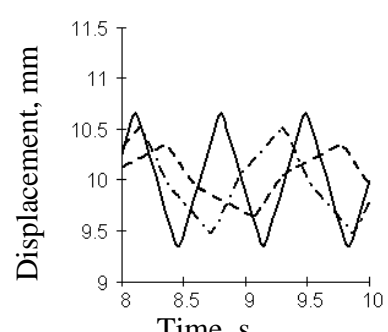

Time, $\mathrm{s}$

a) type of SEO for $x_{2}$

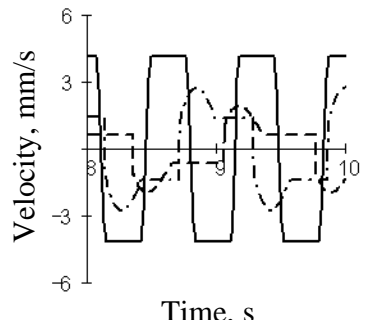

b) type of SEO for $v_{2}$
Fig. 2. Type of SEO in system $I$, depending on the damping coefficient: $\longrightarrow b=0 \mathrm{~N} /(\mathrm{mm} \cdot \mathrm{s}) ;-\bullet-b=200 \mathrm{~N} /(\mathrm{mm} \cdot \mathrm{s})$; - - $b=400 \mathrm{~N} /(\mathrm{mm} \cdot \mathrm{s})$

$$
\left\{\begin{array}{l}
\dot{v}_{1}=\frac{S_{X} \omega_{X X}\left(\frac{u_{\text {yпp }}\left(x_{1}, v_{1}, x_{2}, v_{2}\right)}{U_{0}}-\frac{S_{X}\left[c f_{\Delta}\left(x_{1}-x_{2}\right)+b \dot{f}_{\Delta}\left(x_{1}-x_{2}\right)\right]}{M_{\Pi}}\right)-v_{1}}{T_{1}} ; \\
\dot{v}_{2}=\frac{c f_{\Delta}\left(x_{1}-x_{2}\right)+b \dot{f}_{\Delta}\left(x_{1}-x_{2}\right)-f_{T}\left[v_{2}, c f_{\Delta}\left(x_{1}-x_{2}\right)+b \dot{f}_{\Delta}\left(x_{1}-x_{2}\right)\right]}{m_{2}},
\end{array}\right.
$$

where $S_{X}$ is the kinematic transfer function of the RSM; $\omega_{X X}$ is the motor idling frequency; $u_{\text {упр }}\left(x_{1}, v_{1}, x_{2}, v_{2}\right)$ is the control voltage at the input; $U_{0}$ is the rated voltage; $M_{\Pi}$ is the starting torque; $T_{1}=J_{1} \omega_{X X} / M_{\Pi}$ is the electromechanical time constant; $J_{1}$ is the moment of inertia of the first link; $c$ is the stiffness coefficient; $b$ is the damping coefficient; $m_{2}$ is the mass of the second link. There are two nonlinearities in the model: clearance $f_{\Delta}$ which is modelled by the "imperceptibility zone", and "dry friction" $f_{T}$ with an exponential falling characteristic:

$$
f_{T}(v, F)= \begin{cases}\min \left(F_{\Pi},|F|\right) \operatorname{sign} F, & v=0 ; \\ {\left[F_{\Pi}-\left(F_{\Pi}-F_{\not B}\right) \exp \left(-v_{T} /|v|\right)\right] \operatorname{sign} v,} & v \neq 0 .\end{cases}
$$

The qualitative picture of the self-excited oscillating regimes in system (1) depends on the type and number of nonlinearities. Therefore, the analysis was carried out for three cases: system with a clearance of $\Delta=0,5 \mathrm{~mm}$ and friction-free (system $I$ ); system without clearance with "dry friction" (2) (system II); system with a clearance and "dry friction" (system III). System I operates in stabilization mode $x_{2} \rightarrow x_{0}(=10 \mathrm{~mm}$ in Fig. $2 a)$; systems $I I$ and $I I I$ in stabilization mode of the sliding velocity $v_{2} \rightarrow v_{0}(=1 \ldots 6 \mathrm{~mm} / \mathrm{min}$ in Fig. 3 and 4$)$.

The analysis of the self-excited oscillations occurring in system $I$ showed that sustained relaxational (sawtoothed) oscillations are established when there is not damping (solid curve in Fig. 2a). When the masses are disconnected, their motion continues independently one

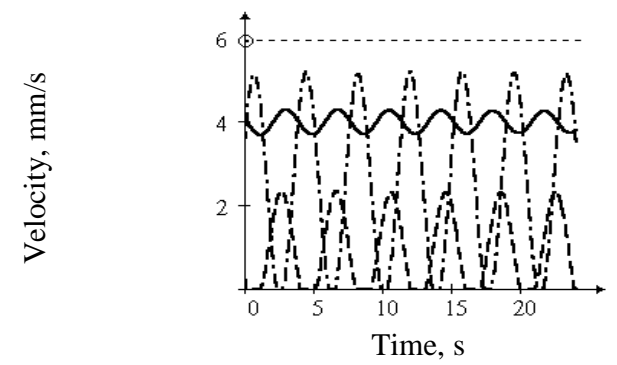

Fig. 3. Type of frictional SEO in system II: - - $1 \mathrm{~mm} / \mathrm{min}$; - $-2,5 \mathrm{~mm} / \mathrm{min} ; \longrightarrow 4 \mathrm{~mm} / \mathrm{min}$;

.. $6 \mathrm{~mm} / \mathrm{min}$ of the other and the load is displaced at the constant velocity that they had at the disconnection time.

During the clearance elimination, the masses collide and they are again disconnected. In a system with damping, the masses can move together after the impact or lose some of their kinetic energy: we can see the sinusoidal zones which correspond to the combined motion of the masses in Fig. $2 b$. Under these conditions, the backlash elimination time is increased due to the lower motion velocity (horizontal sections in Fig. $2 b$ ). The amplitude of the steady-state self-excited oscillations is primarily determined by the clearance size and the damping coefficient. The frequency of the SEO is directly proportional to the stiffness coefficient and inversely proportional to the links mass $\left(J_{1}\right.$ and $\left.m_{2}\right)$ and the damping coefficient.

The task of the control in system $I$ is the fastest backlash elimination in such a way that at the end of the elimination there won't be impact at the joining links time. The algorithm works as follows. Let us suppose that at the moment of the disconnection, the first link was in $\left(x_{1}^{0}, v_{1}^{0}\right)$ state and the second link was in $\left(x_{2}^{0}, v_{2}^{0}\right)$ state. It is necessary to find the trajectory that translates at the shortest time $\tau$ the system from the initial state $\left(x_{1}^{0}, v_{1}^{0}\right)$ to the new state $\left(x_{2}^{0}+v_{2}^{0} \tau \pm \Delta, v_{2}^{0}\right)$ (the sign " \pm " is chosen depending on the sign of the control signal at the disconnection time; to determine it, we assume the signal to be positive). The coordinates of the new state correspond to the position in which the second link moves during the time $\tau$, and the first link should be transferred to the same state. The maximum execution velocity requirement $\tau \rightarrow$ min means that the control signal must be the maximum modulus $\left|u_{\text {упр }}\right|=k U_{0}$, where $k$ is the forcing factor (DBM engines provide a 4...6-fold overload), and the number of voltage changes must be the minimal possible, i.e. one switch or no switching at all. In general, switching is required and the optimal trajectory consists of two sections: (1) forcing the engine with the signal $u_{\text {упр }}=k U_{0}$ before the switching time $\tau^{*}$ and (2) braking the engine by the opposed action mode $u_{\text {упр }}=-k U_{0}$ until the time $\tau$ of the backlash elimination end. The switching time $\tau^{*}$ is determined from the equation of the links velocities at the end of the backlash elimination $\tau: v_{1}(\tau)=v_{2}(\tau)$. The elimination time $\tau$ is implicitly found from the intersection condition of the solutions $x_{1}(\tau)$ and $x_{2}(\tau)=x_{2}^{0}+v_{2}^{0} \tau+\Delta$.

In the numerical study of system $I I$, the following different regimes were qualitatively identified (Fig. 3). At $v>v_{0}(6 \mathrm{~mm} / \mathrm{min})$ there is no self-excited oscillations in the system. If $v_{\mathrm{кр}}<v<v_{0}(4 \mathrm{~mm} / \mathrm{min})$, harmonic frictional SEO are set in the system (without stopping it). Starting with a certain value of the sliding velocity $v \approx v_{\text {кр }}$ $(2,5 \mathrm{~mm} / \mathrm{min})$, the oscillations become relaxational (stopping it). With a further reduction $v<v_{\mathrm{кр}}(1 \mathrm{~mm} / \mathrm{min})$, the stopping time of the output link increases per cycle. 
The frequency of the frictional SEO primarily depends on the load mass and the RSM stiffness. In a mode with a significant stopping rate, a frequency reduction proportional to the increment in the frictional force jump or a sliding velocity reduction is observed.

The dependence on the frictional SEO amplitude is determined by the self-excited oscillating regime: the amplitude is the maximum one in the transitional mode when $v \approx v_{\mathrm{kp}}$. When the self-excited oscillations change as in the harmonic mode as in the relaxational oscillations tightening, the amplitude decreases uniformly to zero. In this sense, we should choose a change in the parameters, which not only reduces the frictional SEO amplitude, but leads the system out of the region of relaxational oscillations. This occurs with a reduction in the frictional force jump and an increment in the sliding velocity, the load mass and the damping.

The algorithm (Fig. 5) to compensate the frictional self-excited oscillations is based on the feedback introduction for the sliding velocity of the output link $v_{2}$. Unlike the linear feedback, when the control voltage is proportional to the magnitude of the disparity, the algorithm works according to the nonlinear law.

1. It is determined and chosen whether the clearance occurs or not The readings of the position sensors $\left(x_{1}^{0}, x_{2}^{0}\right)$ are compared with the condition $\left|x_{1}^{0}-x_{2}^{0}\right|<\Delta_{J}$.

2. The transition time $\tau^{*}$ is calculated from one trajectory to the other. It is determined from the equality of the links velocities at the backlash elimination end time $\tau: v_{1}(\tau)=v_{2}(\tau)$. The elimination time in turn $\tau$ is found from the intersection conditions of the solutions $x_{1}(\tau)$ and $x_{2}(\tau)=x_{2}^{0}+v_{2}^{0} \tau+\Delta_{J}$. (To obtain analytical expressions for $\tau$ and $\tau *$ is impossible, however, their values are unequivocally determined according to the initial values $\left.\left(x_{1}^{0}, v_{1}^{0}, x_{2}^{0}, v_{2}^{0}\right)\right)$. Depending on the magnitudes correlation $\tau$ and $\tau *$, the algorithms continues by one of the three options:

a) if $\tau^{*} \leq 0$, then the program skips step 3 and immediately pass to step 4;

b) if $\tau^{*} \geq \tau$, then only step 3 is executed until the backlash elimination end;

c) if $0<\tau<\tau^{*}$, then step 3 and 4 are sequentially executed.

3. Engine speeding-up (before switching to a different path): while $t<\tau^{*}$, the engine speeding-up is executed by using a control voltage $u_{\text {упр }}=U_{0}$.

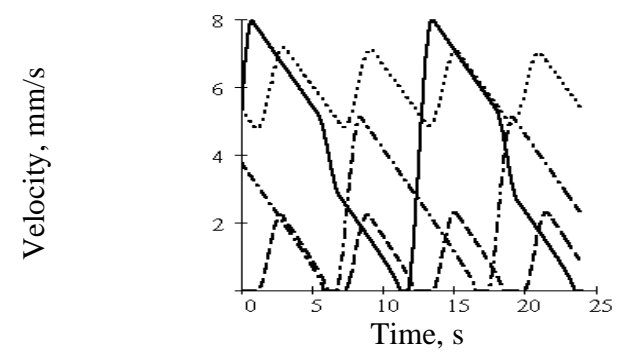

Fig. 4. Type of frictional SEO in system $I I I$ : - $-1 \mathrm{~mm} / \mathrm{min}$; - $-2,5 \mathrm{~mm} / \mathrm{min}$; $4 \mathrm{~mm} / \mathrm{min}$;

.. $6 \mathrm{~mm} / \mathrm{min}$

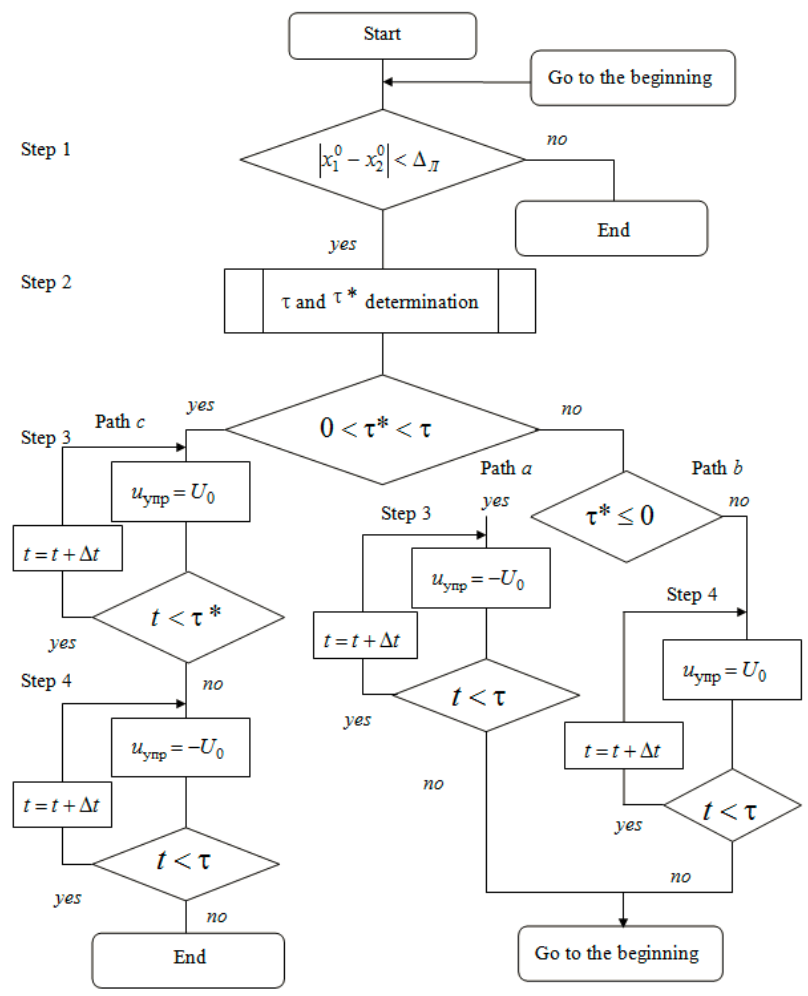

Fig. 5. Algorithm flowchart of the clearance elimination

4. Engine locking (after switching): when $t=\tau^{*}$ swithching is executed to the signal $u_{\text {упр }}=-U_{0}$, which sets the engine locking until the end time $t=\tau$ of the clearance elimination.

If the algorithm past along the path $c$, then, at the backlash elimination time $\tau$, the links have the same speed and no shock occurs. During the execution of the path $a$ or $b$, the drive input link may not be able to reach the required speed $v_{2}^{0}$ at the time $\tau$. Then, during the links engagement, a shock occurs and a new links disconnection is possible. In this case, the program go back to step 1 and determines to chose whether the clearance occurs or not. If necessary, the above steps sequence is repeated.

During the research of a possibility to compensate the clearance influence on the drive output displacement by using digital control, the proposed model did not take into account the delays and errors related to the operation of the microprocessor and as well as the ones introduced by the measuring sensors.

The exact dependencies $u_{\text {упр }}=f\left(v_{2}\right)$ depend on the type of nonlinearity and are too extensive for this publication. The matter of the algorithm can be explained by the example of the stabilization problem $v_{0}=$ const . While $v_{2}=0 \quad u_{\text {упр }}=k U_{0}$. If at the break away torque $v_{2}<v_{0}$, the forcing continues according to the law $u_{\text {упр }}=f_{1}\left(v_{0}-v_{2}\right)$, in that way that after some uniform transitional process $v_{2}=v_{0}$ is established. If at the break away torque $v_{2}>v_{0}$, the engine locking should be carried out by the opposed action mode $u_{\text {упр }}=-f_{2}\left(v_{0}-v_{2}\right)$, and during the velocity reduction till the determined threshold value $v_{2}=v_{0}+\Delta v$, 
switching $u_{\text {yпр }}=f_{3}\left(v_{0}-v_{2}\right)$ occurs again, resulting in an uniform stabilization $v_{2} \leftarrow v_{0}$.

Fig. 4 shows the types of self-excited oscillating regimes in system III with "dry friction" and clearance. In the presence of clearance, the frictional self-excited oscillations become saw-toothed and the value of the critical sliding velocity increases $v_{\text {кр }}$, below which oscillations with stops occur: $v_{\mathrm{rp}}=2,5 \mathrm{~mm} / \mathrm{min}$ in system II, $v_{\text {sp }}=4 \mathrm{~mm} / \mathrm{min}$ in system III. The amplitude and frequency dependences of the frictional SEO on the system parameters qualitatively repeat the regularities noted for the systems $I$ and $I I$.

\section{Conclusion}

The synthesis of the control law is based on the definition of a function $u=u_{\text {ymp }}\left(x_{1}, v_{1}, x_{2}, v_{2}\right)$ that eliminates selfexcited oscillations (or, more formally, minimizes some quality functionality, for example, the accuracy or smoothness of the output displacement, the area of the periodic solution in the phase-plane portrait $\left(x_{2}, v_{2}\right)$, etc.).

The operation design and analysis of the digital control system in a mechatronic drive is most simply and adequately to implement in the specialized dynamic simulation package system Simulink (MATLAB). The modelling capabilities provided by the Simulink environment are diverse; the simulation modelling is undoubtedly one of the possible tools to study dynamic systems.

However, it is important to understand the limitations of its capabilities. Often an important aspect of the research of dynamical systems is the study of the mathematical causes of the appearance of self-excited oscillations and the definition of its bifurcations, where the traditional methods of the qualitative differential equations theory are not replaceable. For example, the definition of the law $u=u_{\text {ymp }}\left(x_{1}, v_{1}, x_{2}, v_{2}\right)$ is realized by the authors in the MathCAD package by using the theory of optimal control resources [11-13].

The proposed algorithm can be implemented in software or tabulated in case of available information on the status and speed of the first and second links. The greatest difficulty is to realize the procedure for determining, determining values $\tau$ and $\tau$ * according to the data $\left(x_{1}^{0}, v_{1}^{0}, x_{2}^{0}, v_{2}^{0}\right)$ from sensors. This procedure solves a system of two transcendental equations with two indeterminates $(\tau$ and $\tau *$ ). In the absence of a kinematic connection the system breaks down into two independent systems that are clearly integrated. The equations form is very extensive to be described here; and the adjustment and optimization of the algorithm has been carried out in the Simulink environment [4].

\section{ACKNOWLEDGMENTS}

The reported study was financially supported according to the scientific research projects 17-20-03084офи_м_РЖД and 9.11716.2018/11.12.

\section{References}

1. Morozov, V.V., Zhdanov, A.V. Peculiarities of the control actuator development on the basis of combined electromechanical modules, Russian Aeronautics 59, 1, pp. 118-125

2. Morozov, V.V., Zhdanov, A.V., Kosterin, A.B. Synthesis of roller screws, Russian Engineering Research 36, 9, pp. 703-707

3. Morozov, V., Fadeev, P., Shtych, D., Belyaev, L., Zhdanov, A. Vibration decrease of electromechanical actuators based on roller screw mechanisms, MATEC Web of Conferences 129, 06026

4. Morozov, V., Zhdanov, A. Self-stopping actuating mechanism with high coefficient of efficiency of the forward stroke for linear motion drives, MATEC Web of Conferences 75 ,09001

5. Karpasyuk V.K., Karpasyuk I.V., Mathematical model of controllable motion of the object in determinate dynamical environment, CASPIAN JOURNAL: Control and High Technologies (PRIKASPIYSKIY ZHURNAL: Upravlenie i Vysokie Tekhnologii), 2012 1, 17, pp. 125-129.

6. Novikova, E., Morozov, V., Zhdanov, A. Development of algorithms for digital control for mechatronic drives consisting of medical devices for rehabilitation, 2015 5th International Workshop on Computer Science and Engineering: Information Processing and Control Engineering, WCSE 2015IPCE.

7. Zhdanov, A., Belyaev, L., Morozov, V. The control system for mechatronic unit of implantable left ventricle assist device, 2016 2nd International Conference on Industrial Engineering, Applications and Manufacturing, ICIEAM 2016, Proceedings 7910951

8. E. Novikova, D. Shtykh, A. Zhdanov, V. Morozov. Increase in accuracy and smoothness of movement of the mechatronic unit of linear micromotions, Applied Mechanics and Materials, 705, 2015, pp 137-141.

9. Zhdanov A.V., Morozov V.V. Theoretical Study of the Load Distribution on the Threads for Roller Screw Mechanisms of a Friction Type, Procedia Engineering, 2016. 150, pp. 992-999.

10. Blinov D, Morozov M, Promising designs of planetary roller screw mechanisms. Izvestiya vuzov. Mishinostroenie 3, 2013, pp. 62-72.

11. Konstantinova, O., Synthesis of new technical decisions based on the object-oriented approach to modelling of technical systems, CASPIAN JOURNAL: Control and High Technologies (PRIKASPIYSKIY ZHURNAL: Upravlenie i Vysokie Tekhnologii), 2010, 3, 11 pp. 26-29.

12. Novikova E. A., Fundaments of the smoothness criteria of mechatronic drives for translational motion, Fundamental research (Fundamentalnye issledovaniya, 2012 3, pp. 123-129 
13. Gusev N. V., Bukreev V. G., Digital control systems for multi-coordinate servo electric drives, Tomsk
Polytechnic University, 2010, 213 p. 